Research Article

\title{
More Constructions of 3-Weight Linear Codes
}

\author{
Lingyong Ma, ${ }^{1}$ Guanjun Li $\mathbb{D},{ }^{2}$ and Fengyan Liu ${ }^{2}$ \\ ${ }^{1}$ College of Computer and Information Sciences, City College of Dongguan University of Technology, Dongguan 523106, \\ Guangdong, China \\ ${ }^{2}$ School of Finance and Mathematics, Huainan Normal University, Huainan 232038, Anhui, China \\ Correspondence should be addressed to Guanjun Li; lichampion2004@126.com
}

Received 31 October 2020; Revised 28 November 2020; Accepted 12 December 2020; Published 4 January 2021

Academic Editor: Ahmed Mostafa Khalil

Copyright (C) 2021 Lingyong Ma et al. This is an open access article distributed under the Creative Commons Attribution License, which permits unrestricted use, distribution, and reproduction in any medium, provided the original work is properly cited.

\begin{abstract}
Linear codes with few weights have become an interesting research topic and important applications of cryptography and coding theory. In this paper, we apply some ternary near-bent and 2-plateaued functions or $r$-ary functions to construct more 3-weight linear codes, where $r$ is a prime. Moreover, we determine the weight distributions of the resulted linear codes by means of some exponential sums.
\end{abstract}

\section{Introduction}

Let $r$ be a prime and $q=r^{m}$. If $\mathscr{C}$ is a $k$-dimensional subspace of $\mathbb{F}_{r}^{n}$, then $\mathscr{C}$ is called an $[n, k]$ linear code over $\mathbb{F}_{r}$. A code $\mathscr{C}$ is called an $[n, k, d]$ code if $d$ is the minimum nonzero weight in $\mathscr{C}$. Let $B_{j}$ be the number of code words of weight $j$ in $\mathscr{C}$. The weight enumerator of $\mathscr{C}$ is defined by

$$
1+B_{1} x+B_{2} x^{2}+\cdots+B_{n} x^{n} .
$$

The list $B_{j}$ for $1 \leq j \leq n$ is called the weight distribution of $\mathscr{C}$. In general, it is difficult to determine the weight distribution of a given linear code $\mathscr{C}$. Information on the weight distribution of linear codes could be seen in [1-9]. If the number of nonzero $B_{j}$ in $\left(1, B_{1}, B_{2}, \ldots, B_{n}\right)$ is equal to $t$, then $\mathscr{C}$ is called a $t$-weight code. Linear codes with few weights have been studied due to their importance in communication and combinatorial theory [10-15]. If the parameters of $\mathscr{C}$ meet a bound on linear codes [16], then $\mathscr{C}$ is called optimal.

For a subset $S=\left\{s_{1}, s_{2}, \ldots, s_{n}\right\}$ of $\mathbb{F}_{q}$, we define

$$
\mathscr{C}_{S}=\left\{c_{a}=\left(\operatorname{tr}\left(a s_{1}\right), \operatorname{tr}\left(a s_{2}\right), \ldots, \operatorname{tr}\left(a s_{n}\right)\right): a \in \mathbb{F}_{q}\right\},
$$

where $\operatorname{tr}(y)=\sum_{i=0}^{s-1} y^{r^{i}}$ is the absolute trace function from $\mathbb{F}_{r^{s}}$ to $\mathbb{F}_{r}$. The set $S$ is called the defining set of $\mathscr{C}_{S}$.

Many linear codes can be generated by choosing the suitable defining set $S[14,17-21]$. In [22], a class of binary linear codes with 3 weights was obtained by using the exponential sum $\sum_{y \in \mathbb{F}_{2 m}}(-1)^{\operatorname{tr}\left(a y^{2^{h}+1}+b y\right)}$. Recently, by using some $r$-ary bent functions, the authors obtained several classes of linear codes with 2 or 3 weights in [23-26].

The Walsh transform of $g(y)$ is defined by

$$
\hat{\chi}_{g}(\lambda)=\sum_{y \in \mathbb{F}_{r^{m}}} \zeta_{r}^{g(y)-\operatorname{tr}(\lambda y)}, \quad \lambda \in \mathbb{F}_{r^{m}}
$$

where $\zeta_{r}=e^{2 \pi \sqrt{-1} / r}$ is a primitive $r$-th root of unity.

Walsh transform is a basic tool to study the properties of cryptography functions. A function $g$ from $\mathbb{F}_{r^{m}}$ to $\mathbb{F}_{r}$ is bent if $\left|\widehat{\chi}_{g}(\lambda)\right|=r^{m / 2}$ for all $\lambda \in \mathbb{F}_{r^{m}}$. For an integer $0 \leq k \leq m, g$ is called $k$-plateaued if $\left|\widehat{\chi}_{g}(\lambda)\right| \in\left\{0, r^{(m+k) / 2}\right\}$ for all $\lambda \in \mathbb{F}_{r^{m}}$. Plateaued function can be used as an ideal candidate function for the design of the cryptographic system because it has a variety of ideal cryptographic characteristics [27].

It should be noted that binary $k$-plateaued functions exist only when $m$ and $k$ have the same parity. The case $k=0$ corresponds to bent functions by definition. 1-plateaued functions are also called near-bent (see [28]), and binary 1plateaued and 2-plateaued functions are called semibent [29].

Based on the aforementioned work, this paper makes further endeavors to construct 3-weight linear codes. We use a class of quadratic ternary near-bent and 2-plateaued functions to construct two classes of ternary 3-weight linear codes. 
Moreover, the weight distributions of the resulted linear codes can be completely given. Numerical results indicate the proposed linear codes contain some linear codes with good parameters. By analyzing the values of some Weil sums, a class of $r$-ary linear codes with 3 weights is presented.

The organization of this paper is as follows. Section 2 gives two classes of ternary linear codes with 3 weights. In Section 3, a class of $r$-ary linear codes with 3 weights is provided by using some Weil sums. Conclusions are finally drawn in Section 4.

\section{Ternary Linear Codes with 3 Weights from Some Ternary Functions}

Throughout this section, let $r$ be an odd prime and $m$ a positive integer. Let $\mathbb{F}_{3^{k}}$ denote the finite field with $3^{k}$ elements and $\mathbb{F}_{3^{k}}^{*}=\mathbb{F}_{3^{k}},\{0\}$. In $[24,26]$, some $r$-ary linear codes with 2 or 3 weights have been obtained by exploiting $r$-ary bent functions. We give two constructions of ternary linear codes with 3 weights by utilizing some ternary functions in this section.

Let $g(x)$ be a function satisfying $g(-x)=g(x)$ and $g(0)=0$. In this section, the defining set $S_{a}$ of $\mathscr{C}_{S_{a}}$ is given by

$$
S_{a}=\left\{x \in \mathbb{F}_{3^{m}}^{*}: g(x)=a\right\},
$$

where $a \in \mathbb{F}_{3}$. Denote $n_{a}=\left|S_{a}\right|$. Clearly, $n_{a}$ is the length $n$ of $\mathscr{C}_{S_{a}}$. We compute

$$
\begin{aligned}
n_{a} & =\frac{1}{3} \sum_{x \in \mathbb{F}_{3^{m}}^{*}} \sum_{y \in \mathbb{F}_{3}} z_{3}^{y(g(x)-a)} \\
& =\frac{1}{3} \sum_{x \in \mathbb{F}_{3^{m}}^{*}}\left(1+\zeta_{3}^{-a} \zeta_{3}^{g(x)}+\zeta_{3}^{a} \zeta_{3}^{-g(x)}\right) \\
& = \begin{cases}3^{m-1}-1+\frac{1}{3}\left(\widehat{\chi}_{g}(0)+\overline{\widehat{\chi}_{g}(0)}\right) & \text { if } a=0, \\
3^{m-1}+\frac{1}{3}\left(\zeta_{3}^{-a} \widehat{\chi}_{g}(0)+\zeta_{3}^{a} \overline{\widehat{\chi}_{g}(0)}\right) & \text { if } a \neq 0 .\end{cases}
\end{aligned}
$$

Note that wt $\left(\mathbf{c}_{b}\right)$ of $\mathbf{c}_{b}$ is $n_{a}-M_{b}$, where

$$
M_{b}=\mid\left\{x \in \mathbb{F}_{3^{m}}^{*}: g(x)=a \text { and } \operatorname{tr}(b x)=0\right\} \mid,
$$

for each $b \in \mathbb{F}_{3^{m}}^{*}$. Note that $f(-x)=f(x)$. We have

$$
\begin{aligned}
& M_{b}=\frac{1}{3^{2}} \sum_{x \in \mathbb{F}_{3 m}^{*}}\left(\sum_{y \in \mathbb{F}_{3}} \zeta_{3}^{y(g(x)-a)}\right)\left(\sum_{z \in \mathbb{F}_{3}} \zeta_{3}^{z \operatorname{tr}(b x)}\right) \\
& =\frac{1}{3^{2}} \sum_{x \in \mathbb{F}_{3}^{*}}\left(1+\zeta_{3}^{-a} \zeta_{3}^{g(x)}+\zeta_{3}^{a} \zeta_{3}^{-g(x)}\right)\left(1+\zeta_{3}^{\operatorname{tr}(b x)}+\zeta_{3}^{\operatorname{tr}(b x)}\right) \\
& =\frac{1}{3^{2}}\left(3^{m}-3-3\left(\zeta_{3}^{-a}+\zeta_{3}^{a}\right)\right)+\frac{1}{3^{2}}\left(\zeta_{3}^{-a} \widehat{\chi}_{g}(0)+\zeta_{3}^{a} \overline{\hat{\chi}_{g}(0)}+2\left(\zeta_{3}^{-a} \widehat{\chi}_{g}(b)+\zeta_{3}^{a} \overline{\hat{\chi}_{g}(b)}\right)\right) \\
& = \begin{cases}3^{m-2}-1+\frac{1}{3^{2}}\left(\widehat{\chi}_{g}(0)+\overline{\hat{\chi}_{g}(0)}+2\left(\hat{\chi}_{g}(b)+\overline{\hat{\chi}_{g}(b)}\right)\right) & \text { if } a=0, \\
3^{m-2}+\frac{1}{3^{2}}\left(\zeta_{3}^{-a} \widehat{\chi}_{g}(0)+\zeta_{3}^{a} \overline{\hat{\chi}_{g}(0)}+2\left(\zeta_{3}^{-a} \widehat{\chi}_{g}(b)+\zeta_{3}^{a} \overline{\hat{\chi}_{g}(b)}\right)\right) & \text { if } a \neq 0 .\end{cases}
\end{aligned}
$$

Generally, it is difficult to give the Walsh coefficients of a ternary function. In the following, we shall select some proper ternary plateaued functions to obtain linear codes $\mathscr{C}_{S_{0}}$ with 3 weights over $\mathbb{F}_{3}$, where $S_{0}$ is defined as in (4).

Lemma 1 (see [30], Theorem 2). Assume that $k>3$, $\lambda, u, v \in \mathbb{F}_{3^{k}}^{*}$, and $\eta$ is the quadratic character of $\mathbb{F}_{3^{k}}$. Define

$$
g(y)=\operatorname{tr}\left(\lambda y^{2}\right)+\operatorname{tr}(u y) \operatorname{tr}(v y)
$$

(i) If $\operatorname{tr}(u v / \lambda)=2, \operatorname{tr}\left(u^{2} / \lambda\right)=1$, and $\operatorname{tr}\left(v^{2} / \lambda\right)=1$, then $g(y)$ is near-bent. Moreover, for $a \in \mathbb{F}_{3^{k}}$,

$$
\hat{\chi}_{g}(a)= \begin{cases}\eta(\lambda)(-1)^{k} i^{k+1} 3^{(k+1) / 2} \zeta_{3}^{-\operatorname{tr}\left(a^{2} / \lambda\right)}, & \text { if }\left(\operatorname{tr}\left(\frac{a u}{\lambda}\right) \operatorname{tr}\left(\frac{a v}{\lambda}\right)\right)=(0,0) \\ \eta(\lambda)(-1)^{k} i^{k+1} 3^{(k+1) / 2} \zeta_{3}^{-\operatorname{tr}\left(a^{2} / \lambda\right)+1}, & \text { if }\left(\operatorname{tr}\left(\frac{a u}{\lambda}\right), \operatorname{tr}\left(\frac{a v}{\lambda}\right)\right)=(1,1) \text { or }(2,2), \\ 0, & \text { otherwise. }\end{cases}
$$


(ii) If $\operatorname{tr}(u v / \lambda)=1, \operatorname{tr}\left(u^{2} / \lambda\right)=0$, and $\operatorname{tr}\left(v^{2} / \lambda\right)=0$, then $g(y)$ is a 2-plateaued function. Moreover, for $a \in \mathbb{F}_{3^{m}}$,

$$
\hat{\chi}_{g}(a)= \begin{cases}\eta(\lambda)(-1)^{k-1} i^{k} 3^{(k / 2)+1} \zeta_{3}^{-\operatorname{tr}\left(a^{2} / \lambda\right)} & \operatorname{if}\left(\operatorname{tr}\left(\frac{a u}{\lambda}\right), \operatorname{tr}\left(\frac{a v}{\lambda}\right)\right)=(0,0), \\ 0 & \text { otherwise. }\end{cases}
$$

The following theorem gives two classes of linear codes with 3 weights over $\mathbb{F}_{3}$.

Theorem 1. Let $k$ be a positive integer with $k>3$ and $\lambda \in \mathbb{F}_{3 k^{*}}$. Let $u, v \in \mathbb{F}_{3^{k}}^{*}$ such that $\operatorname{tr}\left(\lambda^{-1} u v\right)=2, \operatorname{tr}\left(\lambda^{-1} u^{2}\right)=1$, and $\operatorname{tr}\left(\lambda^{-1} v^{2}\right)=1$. Let $g$ be defined as in (8) and $S_{0}$ be defined as in (4).

(i) When $k$ is even, $\mathscr{C}_{S_{0}}$ is a $\left[3^{k-1}-1, k\right]$ code with 3 weights and has weight distribution given in Table 1

(ii) When $k$ is odd, $\mathscr{C}_{S_{0}}$ is a $\left[3^{k-1}+2 \delta \eta(\lambda) 3^{(k-1) / 2}-1, k\right]$ code with 3 weights and has weight distribution given in Table 2, where $\delta=1$ if $k \equiv 1(\bmod 4)$ and $\delta=-1$ if $k \equiv 3(\bmod 4)$

Proof.

Note that $\operatorname{tr}\left(\lambda^{-1} u v\right)=2, \quad \operatorname{tr}\left(\lambda^{-1} u^{2}\right)=1, \quad$ and $\operatorname{tr}\left(\lambda^{-1} v^{2}\right)=1$. By Lemma 1 (i), we have $\hat{\chi}_{g}(0)+\overline{\hat{\chi}}_{g}(0)=$ 0 as $k$ is even. It follows from (5) that $n_{0}=3^{k^{-1}}-1$. Hence, the length $n$ of $\mathscr{C}_{S_{0}}$ is $n_{0}=3^{k-1}-1$.

For $a \in \mathbb{F}_{3 k}^{*}$, to calculate $M_{a}$, we need to calculate $\hat{\chi}_{g}(a)+\overline{\hat{\chi}}_{g}(a)$ from (7).

If $k \equiv 0(\bmod 4), \quad$ then $i^{k+1}=i$. We evaluate $\hat{\chi}_{g}(a)+\overline{\hat{\chi}_{g}(a)}$ by distinguishing the following three cases.

When $(\operatorname{tr}(a u / \lambda), \operatorname{tr}(a v / \lambda))=(0,0)$, it follows from (9) that

$$
\hat{\chi}_{g}(a)+{\overline{\hat{\chi}_{g}(a)}}= \begin{cases}0 & \text { if } \operatorname{tr}\left(\frac{a^{2}}{\lambda}\right)=0, \\ \eta(\lambda) 3^{(k+1) / 2} & \text { if } \operatorname{tr}\left(\frac{a^{2}}{\lambda}\right)=1, \\ -\eta(\lambda) 3^{(k+1) / 2} & \text { if } \operatorname{tr}\left(\frac{a^{2}}{\lambda}\right)=2 .\end{cases}
$$

When $(\operatorname{tr}(a u / \lambda), \operatorname{tr}(a v / \lambda))=(1,1)$ or $(2,2)$, it follows from (9) that

$$
\hat{\chi}_{g}(a)+\overline{\hat{\chi}_{g}(a)}= \begin{cases}-\eta(\lambda) 3^{(k+1) / 2} & \text { if } \operatorname{tr}\left(\frac{a^{2}}{\lambda}\right)=0, \\ \eta(\lambda) 3^{(k+1) / 2} & \text { if } \operatorname{tr}\left(\frac{a^{2}}{\lambda}\right)=1, \\ 0 & \text { if } \operatorname{tr}\left(\frac{a^{2}}{\lambda}\right)=2 .\end{cases}
$$

When $(\operatorname{tr}(a u / \lambda), \operatorname{tr}(a v / \lambda)) \in \mathbb{F}_{3}^{2},\{(0,0),(1,1),(2,2)\}$,

$$
\widehat{\chi}_{g}(a)+\overline{\widehat{\chi}_{g}(a)}=0 .
$$

It then follows from (7) with $b=0$ that

$$
\begin{aligned}
& M_{a} \in\left\{3^{k-2}-1,3^{k-2}-2 \eta(\lambda) 3^{(k-2) / 2}-1,3^{k-2}\right. \\
& \left.\quad+2 \eta(\lambda) 3^{(k-2) / 2}-1\right\} .
\end{aligned}
$$

Hence, the Hamming weight wt $\left(\mathbf{c}_{a}\right)$ of $\mathbf{c}_{a}$ satisfies

$$
\begin{aligned}
\operatorname{wt}\left(\mathbf{c}_{a}\right)= & n_{0}-M_{a} \in\left\{2 \cdot 3^{k-2}-2 \eta(\lambda) 3^{(k-2) / 2}, 2 \cdot 3^{k-2},\right. \\
& \left.2 \cdot 3^{k-2}+2 \eta(\lambda) 3^{(k-2) / 2}\right\} .
\end{aligned}
$$

This implies that the code $\mathscr{C}_{S_{0}}$ has 3 weights in the above set.

If $k \equiv 2(\bmod 4)$, then $i^{k+1}=-i$. Similar to the proof above, we obtain

$$
\begin{aligned}
\operatorname{wt}\left(\mathbf{c}_{a}\right)= & n_{0}-M_{a} \in\left\{2 \cdot 3^{k-2}-2 \eta(\lambda) 3^{(k-2) / 2}, 2 \cdot 3^{k-2},\right. \\
& \left.2 \cdot 3^{k-2}+2 \eta(\lambda) 3^{(k-2) / 2}\right\},
\end{aligned}
$$

for each $a \in \mathbb{F}_{3^{k}}^{*}$. Since $g(-x)=0$ if $g(x)=0$ and $0 \notin S_{0}$, we have the minimum distance of $\mathscr{C}_{S_{0}}^{\perp}$ is 2 . It is easy to see that the number $B_{2}^{\perp}$ of code words with weight 2 in $\mathscr{C}_{S_{0}}^{\perp}$ is $n$. The first three Pless power 
TABLE 1: Weight distribution of $\mathscr{C}_{S_{0}}$ for Theorem 1 (i).

\begin{tabular}{lc}
\hline Weight & Frequency \\
\hline 0 & 1 \\
$2 \cdot 3^{k-2}-2 \eta(\lambda) 3^{(k / 2)-1}$ & $3^{k-2}+\eta(\lambda) 3^{(k / 2)-1}$ \\
$2 \cdot 3^{k-2}$ & $3^{k}-1-2 \cdot 3^{k-2}$ \\
$2 \cdot 3^{k-2}+2 \eta(\lambda) 3^{(k / 2)-1}$ & $3^{k-2}-\eta(\lambda) 3^{(k / 2)-1}$ \\
\hline
\end{tabular}

TABLE 2: Weight distribution of $\mathscr{C}_{S_{0}}$ for Theorem 1 (ii).

\begin{tabular}{lc}
\hline Weight & Frequency \\
\hline 0 & 1 \\
$2 \cdot 3^{k-2}+4 \delta \eta(\lambda) 3^{(k-3) / 2}$ & $2 \cdot 3^{k-1}$ \\
$2 \cdot 3^{k-2}$ & $3^{k-2}+2 \delta \eta(\lambda) 3^{k-3 / 2}-1$ \\
$2 \cdot 3^{k-2}+6 \delta \eta(\lambda) 3^{(k-3) / 2}$ & $2 \cdot 3^{k-2}-2 \delta \eta(\lambda) 3^{(k-3) / 2}$ \\
\hline
\end{tabular}

moments give the desired weight distribution. Since $k>3$, wt $\left(\mathbf{c}_{a}\right)>0$ for any $a \in \mathbb{F}_{3^{k}}^{*}$. Hence, the dimension of $\mathscr{C}_{S_{0}}$ is $k$.

(ii) Conclusion (ii) can be proved by the similar proof as (i).

Example 1. Let $k=4, \lambda=1, u=-1$, and $v=1$. Define $g(y)=\operatorname{tr}\left(y^{2}\right)-\operatorname{tr}(y)^{2}$. Then, $\mathscr{C}_{S_{0}}$ in Theorem 1 (i) is a ternary $[26,4,12]$ code, and its weight enumerator is $1+12 z^{12}+62 z^{18}+6 z^{24}$.

Theorem 2. Let $k$ be a positive integer with $k>4$ and $\lambda \in \mathbb{F}_{3^{k}}^{*}$. Let $u, v \in \mathbb{F}_{3^{k}}$ such that $\operatorname{tr}\left(\lambda^{-1} u v\right)=1, \operatorname{tr}\left(\lambda^{-1} u^{2}\right)=0$, and $\operatorname{tr}\left(\lambda^{-1} v^{2}\right)=0$. Let $S_{0}$ be defined as in (4) and $f$ be defined as in (8).

(i) When $k$ is even, $\mathscr{C}_{S_{0}}$ is a $\left[3^{k-1}-2 \delta \eta(\lambda) 3^{(k / 2)}-1, k\right]$ 3 -weight code and has weight distribution listed in Table 3 , where $\delta=1$ if $k \equiv 0(\bmod 4)$ and $\delta=-1$ if $k \equiv 2(\bmod 4)$

(ii) When $k$ is odd, $\mathscr{C}_{S_{0}}$ is a $\left[3^{k-1}-1, k\right] 3$-weight code and has weight distribution listed in Table 4

Proof. We just omit the proof here since it is similar to that of Theorem 1.

Remark 1. It should be noted that if $\lambda$ is a nonsquare in $\mathbb{F}_{3^{4}}^{*}$, the first conclusion in Theorem 2 is also true when $k=4$.

Example 2. Assume $k=4$ and $\beta$ is the generator of $\mathbb{F}_{3^{4}}^{*}$, where $\beta$ is a root of $x^{4}-x^{3}-1=0$. Let $\lambda=\beta, u=\beta^{16}$, and $v=\beta^{8}$. Define $g(x)=\operatorname{tr}\left(\beta x^{2}\right)+\operatorname{tr}\left(\beta^{16} x\right) \operatorname{tr}\left(\beta^{8} x\right)$. Then, the code $\mathscr{C}_{S_{0}}$ in Theorem 2 (i) is $[44,4,18]$ code and has weight enumerator $1+4 z^{18}+72 z^{30}+4 z^{36}$.

Since $g(x)=g(-x)$, the set $S_{0}$ defined by (4) can be expressed as

$$
S_{0}=-\bar{S} \cup \bar{S},
$$

where $d_{i} d_{j}^{-1} \neq \pm 1$ for $d_{i}, d_{j} \in \bar{S}$ and $d_{i} \neq d_{j}$. Selecting $\bar{D}$ as the defining set can give more ternary 3 -weight codes.
TABLE 3: Weight distribution of $\mathscr{C}_{S_{0}}$ for Theorem 2 (i).

\begin{tabular}{lc}
\hline Weight & Frequency \\
\hline 0 & 1 \\
$2 \cdot 3^{k-2}-4 \delta \eta(\lambda) 3^{(k / 2)-1}$ & $3^{k}-3^{k-2}$ \\
$2 \cdot 3^{k-2}$ & $3^{k-3}-2 \delta \eta(\lambda) 3^{(k / 2)-2}-1$ \\
$2 \cdot 3^{k-2}-6 \delta \eta(\lambda) 3^{(k / 2)-1}$ & $2 \cdot 3^{k-3}+2 \delta \eta(\lambda) 3^{(k / 2)-2}$ \\
\hline
\end{tabular}

TABle 4: Weight distribution of $\mathscr{C}_{S_{0}}$ for Theorem 2 (ii).

\begin{tabular}{lc}
\hline Weight & Frequency \\
\hline 0 & 1 \\
$2 \cdot 3^{k-2}-2 \eta(\lambda) 3^{(k+1 / 2)-1}$ & $3^{k-3}+\eta(\lambda) 3^{(k-3 / 2)}$ \\
$2 \cdot 3^{k-2}$ & $3^{k}-2 \cdot 3^{k-3}-1$ \\
$2 \cdot 3^{k-2}+2 \eta(\lambda) 3^{(k+1 / 2)-1}$ & $3^{k-3}-\eta(\lambda) 3^{(k-3) / 2}$ \\
\hline
\end{tabular}

Corollary 1. Assume that $k>3$ and $\lambda \in \mathbb{F}_{3^{k}}^{*}$. Let $u, v \in \mathbb{F}_{3^{k}}^{*}$ such that $\operatorname{tr}\left(\lambda^{-1} u v\right)=2, \operatorname{tr}\left(\lambda^{-1} u^{2}\right)=1$, and $\operatorname{tr}\left(\lambda^{-1} v^{2}\right)=1$. Let $\bar{D}$ be given as in (17) and $g$ be given as in (8).

(i) When $k$ is even, $\mathscr{C}_{\bar{s}}$ is a $\left[\left(3^{k-1}-1\right) / 2, t k\right] 3$-weight code and has weight distribution given in Table 5

(ii) When $k$ is odd, $\mathscr{C}_{\bar{s}}$ is a $\left[\left(\left(3^{k-1}-1\right) / 2\right)+\right.$ $\left.\delta \eta(\lambda) 3^{(k-1) / 2}, k\right] 3$-weight code and has weight distribution given in Table 6 , where $\delta=1$ if $k \equiv 1(\bmod 4)$ and $\delta=-1$ if $k \equiv 3(\bmod 4)$

Example 3. Let $k=4$. Let $\lambda=1, u=-1$, and $v=1$. Define $g(y)=\operatorname{tr}\left(y^{2}\right)-\operatorname{tr}(y)^{2}$. The code $\mathscr{C}_{\bar{S}}$ in Corollary 1 (i) is a $[13,4,6]$ code and has weight enumerator $1+12 z^{6}+$ $62 z^{9}+6 z^{12}$

Example 4. Let $k=5$. Let $\lambda=-1, u=-1$, and $v=1$. Define $g(y)=\operatorname{tr}\left(-y^{2}\right)-\operatorname{tr}(y)^{2}$. Then, $\mathscr{C}_{\bar{S}}$ in Corollary 1 (ii) is a $[31,5,18]$ code and has weight enumerator $1+60 z^{18}+$ $162 z^{21}+20 z^{27}$. By using the Griesmer bound, we know this code is optimal.

Corollary 2. Assume that $k>4$ and $\lambda \in \mathbb{F}_{3^{k}}^{*}$. Let $u, v \in \mathbb{F}_{3^{k}}^{*}$ such that $\operatorname{tr}\left(\lambda^{-1} u v\right)=1, \operatorname{tr}\left(\lambda^{-1} u^{2}\right)=0$, and $\operatorname{tr}\left(\lambda^{-1} v^{2}\right)=0$. Let $\bar{S}$ be defined as in (17) and $f$ be defined as in (8).

(i) When $k$ is even, $\mathscr{C}_{\bar{s}}$ is a $\left[\left(\left(3^{k-1}-1\right) / 2\right)-\right.$ $\left.\delta \eta(\lambda) 3^{(k / 2)}, k\right] 3$-weight code, and its weight distribution is listed in Table 7 , where $\delta=1$ if $k \equiv 0(\bmod 4)$ and $\delta=-1$ if $k \equiv 2(\bmod 4)$

(ii) When $k$ is odd, $\mathscr{C}_{\bar{S}}$ is a $\left[\left(3^{k-1}-1\right) / 2, k\right] 3$-weight code and has weight distribution listed in Table 8

Example 5. Assume $\beta$ is a generator of $\mathbb{F}_{3^{4}}^{*}$, where $\beta$ is a root of $x^{4}-x^{3}-1=0$. Let $\lambda=\beta, u=\beta^{16}$, and $v=\beta^{8}$. Define $g(y)=\operatorname{tr}\left(\beta y^{2}\right)+\operatorname{tr}\left(\beta^{16} y\right) \operatorname{tr}\left(\beta^{8} y\right)$. Then, the code $\mathscr{C}_{\bar{s}}$ in Corollary 2 (i) is a $[22,4,9]$ code and has weight enumerator $1+4 z^{9}+72 z^{15}+4 z^{18}$. 
TABLe 5: Weight distribution of $\mathscr{C}_{\bar{S}}$ for Corollary 1 (i).

\begin{tabular}{lc}
\hline Weight & Frequency \\
\hline 0 & 1 \\
$3^{k-2}-\eta(\lambda) 3^{(k / 2)-1}$ & $3^{k-2}+\eta(\lambda) 3^{(k / 2)-1}$ \\
$3^{k-2}$ & $3^{k}-1-2 \cdot 3^{k-2}$ \\
$3^{k-2}+\eta(\lambda) 3^{(k / 2)-1}$ & $3^{k-2}-\eta(\lambda) 3^{(k / 2)-1}$ \\
\hline
\end{tabular}

TABLE 6: Weight distribution of $\mathscr{C}_{\bar{S}}$ for Corollary 1 (ii).

\begin{tabular}{lc}
\hline Weight & Frequency \\
\hline 0 & 1 \\
$3^{k-2}+2 \delta \eta(\lambda) 3^{(k-3) / 2}$ & $2 \cdot 3^{k-1}$ \\
$3^{k-2}$ & $3^{k-2}+2 \delta \eta(\lambda) 3^{(k-3) / 2}-1$ \\
$3^{k-2}+3 \delta \eta(\lambda) 3^{(k-3) / 2}$ & $2 \cdot 3^{k-2}-2 \delta \eta(\lambda) 3^{(k-3) / 2}$ \\
\hline
\end{tabular}

TABLe 7: Weight distribution of $\mathscr{C}_{\bar{S}}$ for Corollary 2 (i).

\begin{tabular}{lc}
\hline Weight & Frequency \\
\hline 0 & 1 \\
$3^{k-2}-2 \delta \eta(\lambda) 3^{(k / 2)-1}$ & $3^{k}-3^{k-2}$ \\
$3^{k-2}$ & $3^{k-3}-2 \delta \eta(\lambda) 3^{(k / 2)-2}-1$ \\
$3^{k-2}-3 \delta \eta(\lambda) 3^{(k / 2)-1}$ & $2 \cdot 3^{k-3}+2 \delta \eta(\lambda) 3^{(k / 2)-2}$ \\
\hline
\end{tabular}

TABle 8: Weight distribution of $\mathscr{C}_{\bar{S}}$ for Corollary 2 (ii).

\begin{tabular}{lc}
\hline Weight & Frequency \\
\hline 0 & 1 \\
$3^{k-2}-\eta(\lambda) 3^{((k+1) / 2)-1}$ & $3^{k-3}+\eta(\lambda) 3^{(k-3) / 2}$ \\
$3^{k-2}$ & $3^{m}-2 \cdot 3^{m-3}-1$ \\
$3^{k-2}+\eta(\lambda) 3^{((k+1) / 2)-1}$ & $3^{k-3}-\eta(\lambda) 3^{(k-3) / 2}$ \\
\hline
\end{tabular}

\section{A Class of $r$-Ary Linear Codes with 3 Weights}

Denote $d=\operatorname{gcd}(h, m)$, where $m$ and $h$ are positive integers. For $\lambda, a \in \mathbb{F}_{r^{m}}$, let $f(x)=\operatorname{tr}\left(\lambda x^{r^{h}+1}\right)$.

Based on the fact that a quadratic $r$-ary bent function has full rank, Zhou et al. ([26], Section III.B) obtained a class of linear codes with 2 or 3 weights by using Gold functions. Following the work of Zhou et al. [26], we shall employ Gold class of plateaued functions whose rank is less than $m$ to construct $r$-ary linear codes with 3 weights.

For $\lambda, a \in \mathbb{F}_{r^{m}}$, we denote by $W_{h}(\lambda, a)$ the Weil sum given by

$$
W_{h}(\lambda, a)=\sum_{x \in \mathbb{E}_{r m}} \zeta_{r}^{\operatorname{tr}}\left(\lambda x^{r^{h}+1}+a x\right)
$$

Lemma 2 (see [31], Theorem 4.1). For $m=2 k$, the equation $\lambda^{r^{h}} x^{r^{2 h}}+\lambda x=0$ has solutions in $\mathbb{F}_{r^{m}}^{*}$ if and only if $(\mathrm{m} / \mathrm{d})$ is even and $\lambda^{\left(r^{m}-1\right) /\left(r^{d}+1\right)}=(-1)^{(k / d)}$.

In [31, 32], the following two lemmas studied explicit evaluations of the exponential sum (18).

Lemma 3 (see [31]). Let $(m / d)$ be even with $m=2 k$. Then, $W_{h}(\lambda, 0)=W_{h}(-\lambda, 0)$, and

$$
W_{h}(\lambda, 0)= \begin{cases}r^{k+d} & \text { if } \lambda^{\left(r^{m}-1\right) /\left(r^{d}+1\right)}=(-1)^{(k / d)} \text { and } \frac{k}{d} \text { is odd, } \\ -r^{k+d} & \text { if } \lambda^{\left(r^{m}-1\right) /\left(r^{d}+1\right)}=(-1)^{(k / d)} \text { and } \frac{k}{d} \text { is even. }\end{cases}
$$

Lemma 4 (see [32]). Let $(m / d)$ be even with $m=2 k$. Assume that $g(x)=\lambda^{r^{h}} x^{r^{2 h}}+\lambda x$ is not a permutation polynomial over $\mathbb{F}_{r^{m}}$. Then, for $a \neq 0$, we have $W_{h}(\lambda, a)=0$ unless the equation $g(x)=-a^{r^{h}}$ is solvable. If this equation is solvable, then

$$
W_{h}(\lambda, a)=-(-1)^{(k / d)} r^{k+d} \zeta_{r}^{-\operatorname{tr}\left(\lambda x_{0}^{r^{h}+1}\right)},
$$

where $x_{0}$ is such that $g\left(x_{0}\right)=-a^{r^{h}}$.

Let $(m / d)$ be even with $m=2 k$ and $\lambda \in \mathbb{F}_{r^{m}}^{*}$ such that $\lambda^{\left(r^{m}-1\right) /\left(r^{d}+1\right)}=(-1)^{(k / d)}$. It follows from Lemmas 3 and 4 and the definition of the plateaued function that $f(x)=\operatorname{tr}\left(\lambda x^{r^{h}+1}\right)$ is a $2 d$-plateaued function if $\lambda^{\left(r^{m}-1\right) /\left(r^{d}+1\right)}=(-1)^{(k / d)}$.

Lemma 5. Let $(m / d)$ be even with $m=2 k$ and $\lambda \in \mathbb{F}_{r^{m}}^{*}$ such that $\lambda^{\left(r^{m}-1\right) /\left(r^{d}+1\right)}=(-1)^{(k / d)}$. Then,

$$
\begin{gathered}
W_{h}(\lambda, 0)=W_{h}(2 \lambda, 0)=\cdots=W_{h}((r-1) \lambda, 0) \\
r^{k+d} \quad \text { if } \lambda^{\left(r^{m}-1\right) /\left(r^{d}+1\right)}=(-1)^{(k / d)} \text { and } \frac{k}{d} \text { is odd, } \\
-r^{k+d} \quad \text { if } \lambda^{\left(r^{m}-1\right) /\left(r^{d}+1\right)}=(-1)^{(k / d)} \text { and } \frac{k}{d} \text { is even. }
\end{gathered}
$$

Proof. Since $(m / d)$ is even, $r-1 \mid\left(\left(r^{m}-1\right) /\left(r^{d}+1\right)\right)$. Hence, $(c \lambda)^{\left(r^{m}-1\right) / r^{d}+1}=\lambda^{\left(r^{m}-1\right) / r^{d}+1}=(-1)^{(k / d)}$ for each $c \in \mathbb{F}_{r}^{*}$. The desired result follows from Lemma 3.

Lemma 6. Let $(m / d)$ be even with $m=2 k$ and $\lambda \in \mathbb{F}_{r^{m}}^{*}$ such that $\lambda^{\left(r^{m}-1\right) /\left(r^{d}+1\right)}=(-1)^{(k / d)}$. For $a \in \mathbb{F}_{r^{m}}^{*}$, we have

$$
\sum_{z \in \mathbb{F}_{r}^{*}} \sum_{y \in \mathbb{F}_{r}^{*}} \sum_{x \in \mathbb{E}_{r} m} \zeta_{r}^{\operatorname{tr}\left(y \lambda x^{r^{h}+1}+z a x\right)} \in\left\{0,-(-1)^{(k / d)}(r-1)^{2} r^{k+d},(-1)^{(k / d)} r^{k+d}(r-1)\right\} .
$$

Proof. Since $(m / d)$ is even and $\lambda^{\left(r^{m}-1\right) /\left(r^{d}+1\right)}=(-1)^{(k / d)}$, the equation $\lambda^{r^{h}} x^{r^{2 h}}+\lambda x=0$ is solvable for $x \in \mathbb{F}_{r^{m}}^{*}$ by Lemma 2 .
For $a \in \mathbb{F}_{r^{m}}^{*}$, we claim that the equation $\lambda^{r^{h}} x^{r^{2 h}}+\lambda x+a^{r^{h}}=0$ is solvable if and only if the equation $(y \lambda)^{r^{h}} x^{r^{2 h}}+(y \lambda) x+$ 
$(z a)^{r^{h}}=0$ is solvable for each $(y, z) \in \mathbb{F}_{r}^{*} \times \mathbb{F}_{r}^{*}$. Suppose $x_{0}$ is a solution of $\lambda^{r^{h}} x^{r^{2 h}}+\lambda x+a^{r^{h}}=0$. Clearly, $x_{0}$ is also a root of $y^{-1} z \lambda^{r^{h}} x^{r^{2 h}}+y^{-1} z \lambda x+y^{-1} z a^{r^{h}}=0$. Then, $y^{-1} z \lambda^{r^{h}} x_{0}^{r^{2 h}}+y^{-1} z \lambda x_{0}+y^{-1} z a^{r^{h}}=0$. We can rewrite this as $\lambda^{r^{h}}\left(y^{-1} z x_{0}\right)^{r^{2 h}}+\lambda\left(y^{-1} z x_{0}\right)+y^{-1} z a^{r^{h}}=0$ since $z, y \in \mathbb{F}_{r}^{*}$. It then follows that $(y \lambda)^{r^{h}}\left(y^{-1} z x_{0}\right)^{r^{2 h}}+(y \lambda)\left(y^{-1} z x_{0}\right)+(z a)^{r^{h}}=0$. That is to say, the equation $(y \lambda)^{r^{h}} x^{r^{2 h}}+(y \lambda) x+(z a)^{r^{h}}=0$ has a solution $y^{-1} z x_{0}$. Conversely, assume $x_{0}$ is a solution of $(y \lambda)^{r^{h}} x^{r^{2 h}}+(y \lambda) x+(z a)^{r^{h}}=0$; then, $(y \lambda)^{r^{h}} x_{0}^{r^{h}}+(y \lambda) x_{0}+(z a)^{r^{h}}=0$. Note that $y, z \in \mathbb{F}_{r}^{*}$.
Hence, we have $\lambda^{r^{h}}\left(z^{-1} y x_{0}\right)^{r^{2 h}}+\lambda\left(z^{-1} y x_{0}\right)+a^{r^{h}}=0$. This implies that $\lambda^{r^{h}} x^{r^{2 h}}+\lambda x+a^{r^{h}}=0$ has a solution $z^{-1} y x_{0}$.

If $\lambda^{r^{h}} x^{r^{2 h}}+\lambda x+a^{r^{h}}=0$ has no solutions in $\mathbb{F}_{r^{m}}$, then by Lemma 4 and the discussions above, we have

$$
\sum_{y \in \mathbb{F}_{r}^{*}} \sum_{z \in \mathbb{F}_{r}^{*}} \sum_{x \in \mathbb{E}_{r} m} \zeta_{r}^{\operatorname{tr}}\left(y \lambda x^{r^{h}+1}+z a x\right)=0 .
$$

If $\lambda^{r^{h}} x^{r^{2 h}}+\lambda x+a^{r^{h}}=0$ has a solution $x_{0}$ in $\mathbb{F}_{r^{m}}$, then we know that $(y \lambda)^{r^{h}} x^{r^{2 h}}+(y \lambda) x+(z a)^{r^{h}}=0$ has a solution $y^{-1} z x_{0}$ from the discussions above. It follows from (20) that

$$
\begin{aligned}
\sum_{z \in \mathbb{F}_{r}^{*}} \sum_{y \in \mathbb{F}_{r}^{*}} \sum_{x \in \mathbb{F}_{r} m} \zeta_{r}^{\operatorname{tr}\left(y \lambda x^{r^{h}+1}+z a x\right)} & =(-1)^{(k / d)+1} r^{k+d} \sum_{z \in \mathbb{F}_{r}^{*}} \sum_{y \in \mathbb{F}_{r}^{*}} \zeta_{r}^{\operatorname{tr}\left(y \lambda\left(y^{-1} z x_{0}\right)^{r^{h}+1}\right)} \\
& =(-1)^{(k / d)+1} r^{k+d} \sum_{z \in \mathbb{F}_{r}^{*}} \sum_{y \in \mathbb{F}_{r}^{*}} \zeta_{r}^{-y^{-1} z^{2} \operatorname{tr}\left(\lambda x_{0}^{h^{h}+1}\right)} \\
& = \begin{cases}(-1)^{(k / d)} r^{k+d}(r-1) & \text { if } \operatorname{tr}\left(\lambda x_{0}^{r^{h}+1}\right) \neq 0, \\
(-1)^{(k / d)+1} r^{k+d}(r-1)^{2} & \text { if } \operatorname{tr}\left(\lambda x_{0}^{r^{h}+1}\right)=0 .\end{cases}
\end{aligned}
$$

Hence, for any $a \in \mathbb{F}_{r^{m}}^{*}$,

$$
\sum_{z \in \mathbb{F}_{r}^{*}} \sum_{y \in \mathbb{F}_{r}^{*}} \sum_{x \in \mathbb{F}_{r^{m}}} \zeta_{r}^{\operatorname{tr}\left(y \lambda x^{r^{h}+1}+z a x\right)} \in\left\{0,(-1)^{(k / d)} r^{k+d}(r-1),(-1)^{(k / d)+1} r^{k+d}(r-1)^{2}\right\}
$$

Let $h$ be a positive integer, $1 \leq h<k$, where $m=2 k>4$ is an even integer. Define

$$
S=\left\{x \in \mathbb{F}_{r^{m}}^{*}: \operatorname{tr}\left(\lambda x^{r^{h}+1}\right)=0\right\}
$$

where $\lambda \in \mathbb{F}_{r^{m}}^{*}$.

Theorem 3. Let $h$ be a positive integer, $1 \leq h<k$, where $m=$ $2 k>4$ is an even integer. Let $S$ be defined as in (26), where $\lambda \in \mathbb{F}_{r^{m}}^{*}$. Define $d=\operatorname{gcd}(h, m)$. Assume that $(m / d)$ is even and $\lambda^{\left(r^{m}-1\right) /\left(r^{d}+1\right)}=(-1)^{(k / d)}$. (i) If $(k / d)$ is odd, then $\mathscr{C}_{S}$ is a $\left[(r-1) r^{k+d-1}+r^{m-1}-\right.$ $\left.1, m, r^{m-2}(r-1)\right]$ 3-weight code and has weight distribution given in Table 9

(ii) If $(k / d)$ is even, then $\mathscr{C}_{S}$ is a $\left[(r-1) r^{k+d-1}-r^{m-1}-\right.$ $\left.1, m, r^{m-2}(r-1)-(r-1) r^{k+d-1}\right] 3$-weight code with the weight distribution given in Table 10

Proof. We only present the proof of (i) since the others can be proven by a similar proof. Denote $n_{S}=|S \cup\{0\}|$. We have

$$
\begin{aligned}
n_{S} & =\frac{1}{r} \sum_{x \in \mathbb{E}_{r} m} \sum_{y \in \mathbb{F}_{r}} \zeta_{r}^{y \operatorname{tr}\left(\lambda x^{r^{h}+1}\right)} \\
& =\frac{1}{r} \sum_{x \in \mathbb{E}_{r m}}\left(1+\zeta_{r}^{\operatorname{tr}\left(\lambda x^{h^{h}+1}\right)}+\zeta_{r}^{2 \operatorname{tr}\left(\lambda x^{r^{h}+1}\right)}+\cdots+\zeta_{r}^{\left.(r-1) \operatorname{tr}\left(\lambda x^{r^{h}+1}\right)\right)}\right. \\
& =r^{m-1}+\frac{1}{r}\left(W_{h}(\lambda, 0)+W_{h}(2 \lambda, 0)+\cdots+W_{h}((r-1) \lambda, 0)\right) .
\end{aligned}
$$

Note that $(k / d)$ is odd and $\lambda^{\left(r^{m}-1\right) /\left(r^{d}+1\right)}=(-1)^{(k / d)}$. By Lemma 5, we have

$$
n=n_{S}-1=r^{m-1}+(r-1) r^{k+d-1}-1
$$


TABle 9: Weight distribution of $\mathscr{C}_{S}$ for Theorem 3 (i).

\begin{tabular}{lr}
\hline Weight & Frequency \\
\hline 0 & 1 \\
$r^{m-2}(r-1)$ & $\left(\left(r^{m-2}-r^{k+d-2}\right)\left(r^{m-2}+r^{k+d-1}\right)\right) /\left(r^{m+2 d-3}\right)$ \\
$r^{m-2}(r-1)+(r-1)^{2} r^{k+d-2}$ & $r^{m}-1-\left(\left(r^{m-2}-r^{k+d-2}\right)\left(r^{m-1}+r^{k+d-1}\right) / r^{m+2 d-3}\right)$ \\
$r^{m-2}(r-1)+(r-1) r^{k+d-1}$ & $\left(\left(r^{m-2}-r^{k+d-2}\right)(r-1) r^{m-2}\right) /\left(r^{m+2 d-3}\right)$ \\
\hline
\end{tabular}

Table 10: Weight distribution of $\mathscr{C}_{S}$ for Theorem 3 (ii).

\begin{tabular}{lr}
\hline Weight & Frequency \\
\hline 0 & 1 \\
$r^{m-2}(r-1)-(r-1) r^{k+d-1}$ & $\left(\left(r^{m-2}+r^{k+d-2}\right)(r-1) r^{m-2}\right) /\left(r^{m+2 d-3}\right)$ \\
$r^{m-2}(r-1)-(r-1)^{2} r^{k+d-2}$ & $r^{m}-1-\left(\left(\left(r^{m-2}+r^{k+d-2}\right)\left(r^{m-1}-r^{k+d-1}\right)\right) /\left(r^{m+2 d-3}\right)\right)$ \\
$r^{m-2}(r-1)$ & $\left(\left(r^{m-2}+r^{k+d-2}\right)\left(r^{m-2}-r^{k+d-1}\right)\right) /\left(r^{m+2 d-3}\right)$ \\
\hline
\end{tabular}

For each $a \in \mathbb{F}_{r^{m}}^{*}$, let

$$
M_{a}=\mid\left\{x \in \mathbb{F}_{r^{m}}: \operatorname{tr}\left(\lambda x^{r^{h}+1}\right)=0 \text { and } \operatorname{tr}(a x)=0\right\} \mid \text {. }
$$

Then, the Hamming weight wt $\left(\mathbf{c}_{a}\right)$ is $n_{S}-M_{a}$. We can compute

$$
\begin{aligned}
M_{a} & =\frac{1}{r^{2}} \sum_{x \in \mathbb{F}_{r} m}\left(\sum_{y \in \mathbb{F}_{r}} \zeta_{r}^{y \operatorname{tr}\left(\lambda x^{r^{h}+1}\right)}\right)\left(\sum_{z \in \mathbb{F}_{r}} \zeta_{r}^{z \operatorname{tr}(a x)}\right) \\
& =r^{m-2}+\frac{1}{r^{2}}\left(\sum_{y \in \mathbb{F}_{r}^{*}} \sum_{x \in \mathbb{F}_{r} m} \zeta_{r}^{y \operatorname{tr}\left(\lambda x^{r^{h}+1}\right)}\right)+\frac{1}{r^{2}} \sum_{y \in \mathbb{F}_{r}^{*}} \sum_{z \in \mathbb{F}_{r}^{*}} \sum_{x \in \mathbb{F}_{r} m} \zeta_{r}^{\operatorname{tr}\left(y \lambda x^{r^{h}+1}+z a x\right)} \\
& =r^{m-2}+\frac{1}{r^{2}}\left(\sum_{y \in \mathbb{F}_{r}^{*}} W_{h}(y \lambda, 0)\right)+\frac{1}{r^{2}} \sum_{y \in \mathbb{F}_{r}^{*}} \sum_{z \in \mathbb{F}_{r}^{*}} \sum_{x \in \mathbb{F}_{r} m} \zeta_{r}^{\operatorname{tr}\left(y \lambda x^{r^{h}+1}+z a x\right)}
\end{aligned}
$$

It follows from Lemmas 5 and 6 that

$$
M_{a} \in\left\{r^{m-2}+(r-1)^{2} r^{k+d-2}, r^{m-2}, r^{m-2}+(r-1) r^{k+d-2}\right\} \text {. }
$$

For each $a \in \mathbb{F}_{r^{m}}^{*}$, we have

$$
\operatorname{wt}\left(\mathbf{c}_{a}\right)=\left\{r^{m-2}(r-1),(r-1) r^{m-2}+(r-1) r^{k+d-1}, r^{m-2}(r-1)+(r-1)^{2} r^{k+d-2}\right\} \text {. }
$$

Then, $\mathscr{C}_{S}$ has three nonzero weights above. When $m>4$, for each $a \in \mathbb{F}_{3^{m}}^{*}$, wt $\left(\mathbf{c}_{a}\right)>0$, and thus, the dimension of the code is $m$.

Note that $0 \notin S$. The minimum distance of $\mathscr{C}_{S}^{\perp}$ cannot be one. Since $\operatorname{tr}\left(\lambda(c x)^{r^{h}+1}\right)=0$ for all $c \in \mathbb{F}_{r}^{*}$, if $\operatorname{tr}\left(\lambda x^{r^{h}+1}\right)=0$, the minimum Hamming weight of $\mathscr{C}_{S}^{\perp}$ is 2 . Moreover, we can prove that $B_{2}^{\perp}$ is equal to $n\left(\begin{array}{c}r-1 \\ 2\end{array}\right)$. In fact, for each $r-1$ distinct element $v, 2 v, \ldots,(r-1) v \in S$, we can get $(r-1)\left(\begin{array}{c}r-1 \\ 2\end{array}\right)$ code words with weight 2 in $\mathscr{C}_{S}^{\perp}$. Define $w_{1}=r^{m-2}(r-1), \quad w_{2}=r^{m-2}(r-1)+r^{k+d-2}(r-1)^{2}$, and $w_{3}=r^{m-2}(r-1)+r(r-1) r^{k+d-2}$.

Solving the following linear equations gives the desired weight distribution: 


$$
\left\{\begin{array}{l}
B_{w_{1}}+B_{w_{2}}+B_{w_{3}}=r^{m}-1, \\
w_{1} B_{w_{1}}+w_{2} B_{w_{2}}+w_{3} B_{w_{3}}=(r-1) n r^{m-1}, \\
w_{1}^{2} B_{w_{1}}+w_{2}^{2} B_{w_{2}}+w_{3}^{2} B_{w_{3}}=\left[(r-1) n((r-1) n+1)+2\left(\begin{array}{c}
r-1 \\
2
\end{array}\right) n\right] r^{m-2} .
\end{array}\right.
$$

Example 6. Let $m=8$ and $h=2$. Let $f(x)=\operatorname{tr}\left(x^{10}\right)$. Then, the code $\mathscr{C}_{S}$ is a $[1700,8,972]$ code and has weight enumerator $1+60 z^{972}+6480 z^{1134}+20 z^{1458}$.

Example 7. Let $m=6$ and $h=1$. Let $\beta$ be the generator of $\mathbb{F}_{5^{6}}^{*}$, where $\beta$ is a root of $x^{6}+x^{4}-x^{3}+x^{2}+2=0$. Let $\lambda=\beta^{3}$. Clearly, $\lambda^{\left(5^{6}-1\right) / 6}=\beta^{7812}=-1$. Let $f(x)=\operatorname{tr}\left(\beta^{3} x^{6}\right)$. Then, the code $\mathscr{C}_{S}$ is a $[3624,6,2500]$ code and has weight enumerator $1+144 z^{2500}+15000 z^{2900}+480 z^{3000}$.

\section{Conclusion}

We succeed in constructing some linear codes with 3 weights from some $r$-ary functions with few Walsh transform values in the odd characteristic. The weight distributions of linear codes in this paper are determined by analyzing Walsh coefficients of $r$-ary functions. Compared with other linear codes with 3 weights, the parameters of the linear codes with 3 weights of this paper are different from those obtained from bent functions in $[19,23,24,26]$. As applications, some of the 3-weight codes can be used to obtain secret sharing schemes [15] and association schemes introduced in [11].

\section{Data Availability}

All datasets generated for this study are included within the article.

\section{Conflicts of Interest}

The authors declare that they have no conflicts of interest.

\section{Acknowledgments}

This work was supported by the Dongguan Science and Technology Development Foundation (Grant no. 2019507151569), the NNSF of China (Grant no. 11601177), and the Natural Science Foundation for the Higher Education Institutions of Anhui Province of China (Grant no. KJ2018A0470).

\section{References}

[1] B. Courteau and J. Wolfmann, "On triple-sum-sets and two or three weights codes," Discrete Mathematics, vol. 50, pp. 179-191, 1984.

[2] C. Ding, C. Li, N. Li, and Z. Zhou, "Three-Weight cyclic codes and their weight distributions," Discrete Mathematics, vol. 339, 2016.

[3] C. Ding, J. Luo, and H. Niederreiter, "Two weight codes punctured from irreducible cyclic codes," in Proceedings of the 1st International Workshop Coding Theory Cryptography, pp. 119-124, Singapore, June 2008.

[4] K. Feng and J. Luo, "Value distributions of exponential sums from perfect nonlinear functions and their applications," IEEE Transactions on Information Theory, vol. 53, no. 9, pp. 3035-3041, 2007.

[5] C. Li, Q. Yue, and F. Li, "Hamming weights of the duals of cyclic codes with two zeros," IEEE Transactions on Information Theory, vol. 60, no. 7, pp. 3895-3902, 2014.

[6] C. Ma, L. Zeng, Y. Liu, D. Feng, and C. Ding, "The weight enumerator of a class of cyclic codes," IEEE Transactions on Information Theory, vol. 57, no. 1, pp. 397-402, 2011.

[7] G. Xu, X. Cao, and S. Xu, "Optimal p-ary cyclic codes with minimum distance four from monomials," Cryptography and Communications, vol. 8, no. 4, pp. 541-554, 2016.

[8] X. Zeng, L. Hu, W. Jiang, Q. Yue, and X. Cao, "The weight distribution of a class of p-ary cyclic codes," Finite Fields and Their Applications, vol. 16, no. 1, pp. 56-73, 2010.

[9] D. Zheng, X. Wang, X. Zeng, and L. Hu, "The weight distribution of a family of $\$ \$ p \$ \$ p$-ary cyclic codes," Designs, Codes and Cryptography, vol. 75, no. 2, pp. 263-275, 2015.

[10] R. Anderson, C. Ding, T. Helleseth, and T. Kløve, "“How to build robust shared control systems," Designs," Codes Cryptography, vol. 15, no. 2, pp. 111-124, 1998.

[11] A. R. Calderbank and J. M. Goethals, "Three-weight codes and association schemes," Philips Journal of Research, vol. 39, pp. 143-152, 1984.

[12] R. Calderbank and W. M. Kantor, "The geometry of twoweight codes," Bulletin of the London Mathematical Society, vol. 18 , no. 2, pp. 97-122, 1986.

[13] C. Carlet, C. Ding, and J. Yuan, "Linear codes from perfect nonlinear mappings and their secret sharing schemes," IEEE Transactions on Information Theory, vol. 51, no. 6, pp. 2089-2102, 2005.

[14] C. Ding and X. Wang, "A coding theory construction of new systematic authentication codes," Theoretical Computer Science, vol. 330, no. 1, pp. 81-99, Jan. 2005.

[15] J. Yuan and C. Ding, "Secret sharing schemes from three classes of linear codes," IEEE Transactions on Information Theory, vol. 52, no. 1, pp. 206-212, 2006.

[16] W. C. Huffman and V. Pless, Fundamentals of Error-Correcting Codes, Cambridge University Press, Cambridge, UK., 2003.

[17] C. Ding and H. Niederreiter, "Cyclotomic linear codes of order \$3," IEEE Transactions on Information Theory, vol. 53, no. 6, pp. 2274-2277, 2007.

[18] C. Ding, J. Luo, and H. Niederreiter, "Two weight codes punctured from irreducible cyclic codes," in Proceedings of the First International Workshop on Coding Theory and Cryptography, Singapore, June 2008.

[19] C. Ding, "Linear codes from some 2-designs," IEEE Transactions on Information Theory, vol. 61, no. 6, pp. 3265-3275, June 2015. 
[20] Z. Heng and Q. Yue, "A class of binary linear codes with at most three weights," IEEE Communications Letters, vol. 19, no. 9, pp. 1488-1491, September 2015.

[21] C. Xiang, "Linear codes from a generic construction," Cryptography and Communications, vol. 8, no. 4, pp. 525-539, 2016.

[22] K. Ding and C. Ding, "Binary linear codes with three weights," IEEE Communications Letters, vol. 18, no. 11, pp. 1879-1882, 2014.

[23] K. Ding and C. Ding, "A class of two-weight and three-weight codes and their applications in secret sharing," IEEE Transactions on Information Theory, vol. 61, no. 11, pp. 5835-5842, 2015.

[24] C. Tang, N. Li, Y. Qi, Z. Zhou, and T. Helleseth, "Linear codes with two or three weights from weakly regular bent functions," IEEE Transactions on Information Theory, vol. 62, no. 3, pp. 1166-1176, 2016.

[25] G. Xu, L. Qu, and X. Cao, "Three classes of minimal linear codes over the finite fields of odd characteristic," IEEE Transactions on Information Theory, vol. 65, no. 11, pp. 7067-7078, 2019.

[26] Z. Zhou, N. Li, C. Fan, and T. Helleseth, "Linear codes with two or three weights from quadratic bent functions," Designs, Codes and Cryptography, vol. 81, no. 2, pp. 283-295, 2016.

[27] Y. Zheng and X.-M. Zhang, "Plateaued functions," Information and Communication Security, vol. 1726, pp. 284-300, 1999.

[28] A. Çeşmelioğlu, G. McGuire, and W. Meidl, "A construction of weakly and non-weakly regular bent functions," Journal of Combinatorial Theory, Series A, vol. 119, pp. 420-429, 2012.

[29] S. Mesnager, "On semi-bent functions and related plateaued functions over the galois field $\mathbb{F}_{2^{n}}$," in Open Problems in Mathematics and Computational Science, Springer, Berlin, Germany, 2013.

[30] G. Xu, X. Cao, and S. Xu, "Several classes of quadratic ternary bent, near-bent and 2-plateaued functions," International Journal of Foundations of Computer Science, vol. 28, no. 01, pp. 1-18, 2017.

[31] R. Coulter, "Explicit evaluations of some Weil sums," Acta Arithmetica, vol. 83, no. 3, pp. 241-251, 1998.

[32] R. Coulter, "Further evaluations of Weil sums," Acta Arithmetica, vol. 86, no. 3, pp. 217-226, 1998. 